\title{
Adaptation effects in static postural control by providing simultaneous visual feedback of center of pressure and center of gravity
}

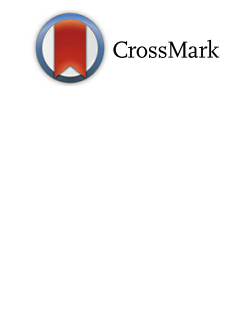

\author{
Kenta Takeda', Hiroki Mani², Naoya Hasegawa', Yuki Sato', Shintaro Tanaka', Hiroshi Maejima²
} and Tadayoshi Asaka ${ }^{2^{*}}$

\begin{abstract}
Background: The benefit of visual feedback of the center of pressure (COP) on quiet standing is still debatable. This study aimed to investigate the adaptation effects of visual feedback training using both the COP and center of gravity (COG) during quiet standing.

Methods: Thirty-four healthy young adults were divided into three groups randomly (COP $+C O G$, COP, and control groups). A force plate was used to calculate the coordinates of the COP in the anteroposterior (COPAP) and mediolateral $\left(\mathrm{COP}_{\mathrm{ML}}\right)$ directions. A motion analysis system was used to calculate the coordinates of the center of mass (COM) in both directions (COM $\mathrm{CP}_{\mathrm{P}}$ and $\left.\mathrm{COM}_{\mathrm{ML}}\right)$. The coordinates of the $\mathrm{COG}$ in the AP direction $\left(C_{\mathrm{AP}}\right)$ were obtained from the force plate signals. Augmented visual feedback was presented on a screen in the form of fluctuation circles in the vertical direction that moved upward as the COPAP and/or $C_{\text {APG }}$ moved forward and vice versa. The COP + COG group received the real-time $C O P_{A P}$ and $C_{\text {AP }}$ feedback simultaneously, whereas the $\mathrm{COP}$ group received the real-time $\mathrm{COP}_{\mathrm{AP}}$ feedback only. The control group received no visual feedback. In the training session, the $C O P+C O G$ group was required to maintain an even distance between the $C O P_{A P}$ and $C O G_{A P}$ and reduce the $\mathrm{COG}_{\mathrm{AP}}$ fluctuation, whereas the COP group was required to reduce the $\mathrm{COP}_{\mathrm{AP}}$ fluctuation while standing on a foam pad. In test sessions, participants were instructed to keep their standing posture as quiet as possible on the foam pad before (pre-session) and after (post-session) the training sessions.
\end{abstract}

Results: In the post-session, the velocity and root mean square of $C O M_{A P}$ in the $C O P+C O G$ group were lower than those in the control group. In addition, the absolute value of the sum of the COP - COM distances in the $\mathrm{COP}+\mathrm{COG}$ group was lower than that in the COP group. Furthermore, positive correlations were found between the $\mathrm{COM}_{\mathrm{AP}}$ velocity and $\mathrm{COP}$ - COM parameters.

Conclusions: The results suggest that the novel visual feedback training that incorporates the $\mathrm{COP}_{\mathrm{AP}}-\mathrm{COG}_{\mathrm{AP}}$ interaction reduces postural sway better than the training using the $\mathrm{COP}_{\mathrm{AP}}$ alone during quiet standing. That is, even $\mathrm{COP}_{\mathrm{AP}}$ fluctuation around the $\mathrm{COG}_{\mathrm{AP}}$ would be effective in reducing the $\mathrm{COM}_{\mathrm{AP}}$ velocity.

Keywords: Center of gravity, Center of mass, Center of pressure, Static posture, Visual feedback training

\footnotetext{
* Correspondence: ask-chu@hs.hokudai.ac.jp

${ }^{2}$ Department of Rehabilitation Science, Faculty of Health Sciences, Hokkaido

University, N12-W5, Kita-ku, Sapporo, Hokkaido 060-0812, Japan

Full list of author information is available at the end of the article
} 


\section{Introduction}

The ability to maintain balance in static postures relies on the ability of the central nervous system to control movements or positional fluctuations by using the body's center of mass (COM) so that it remains within safe boundaries above the base of support $[1,2]$. If static balance during quiet standing is controlled by shifting the center of pressure (COP) through the feet, the body will move as a single segment, often modeled as an inverted pendulum [3]. Previous studies have reported that the augmented visual feedback of the COP has been used for static balance training [4-6].

However, the benefits gained from visual feedback of the COP during quiet standing are still under debate [7, $8]$. Kilby et al. reported that the real-time visual feedback of neither the COP nor the COM affected the postural motion of healthy adults during quiet standing [9]; in other words, neither the COP nor the COM velocities changed when conditions were altered between a presence and lack of augmented visual feedback. In addition, the participants in the study of Lakhani et al. showed no postural stability learning effects when visual feedback training was using either as a vertical projection of the COM onto the ground (i.e., the center of gravity (COG)) or the COP during quiet standing [10]. In fact, under no feedback conditions did the root mean square values of the COP or COG change between the pre-training and post-training sessions.

The impact of the difference in position between the COP and COG (COP-COG distance) on postural stability in static balance has also been investigated [11]. A larger COP-COG distance has been shown to indicate greater body acceleration during quiet standing [12, 13]. During quiet standing, the COP-COG distance increases with age and depends on whether the participant's eyes are closed or open $[12,14]$. Postural instability may result from biased positioning of the COP relative to the COG, as this will result in a unidirectional moment acting on the COM [3]. In fact, Mani et al. reported that elderly participants were not able to maintain equilibrium standing on one leg when the position of the COP was biased relative to the COG, such as when the COP was located laterally in the direction of the supporting leg, while the younger group of participants experienced less difficulty [14]. In addition,
Ibuki et al. reported that the COP - COM distance decreased and the COP fluctuated more evenly around the COM during one-legged standing in the ballet dancer group than in the control group [15]. These findings imply that feedback training incorporating the interaction between the COP and COG may be more effective for improving static balance than training using the COP or COG alone.

With this background in mind, the purpose of this study was to investigate the adaptive effects of augmented visual feedback training using both the COP and COG, as compared to training using only the COP, during quiet standing. The hypothesis was that novel balance training, which incorporates the interaction between the COP and COG using simultaneously the visual feedback of both, would reduce postural sway compared to training that used the COP alone without feedback. The findings of this study could contribute toward the development of an effective visual feedback training system for improving postural static balance.

\section{Methods \\ Participants}

Thirty-four healthy young adults without any known neurological, motor, or visual disorders or disabilities participated in this study. All the study protocols were approved by the ethics committee of the institution where the study was conducted, and written informed consent was obtained from all participants according to the Declaration of Helsinki. The age, sex, height, body weight, and foot length of each participant were recorded (Table 1). The participants were divided randomly into three groups. The first group, i.e., the COP group, received visual feedback of their real-time COP during the training session. The second group, i.e., the COP + COG group, received real-time visual feedback of their COG as well as their COP during the training session. The third group, i.e., the control group, received no visual feedback and was instructed to focus on a fixed visual target.

\section{Equipment}

Kinematic data were collected using a six-camera 3D motion analysis system (Motion Analysis Corporation, Santa Rosa, CA, USA) at a sampling frequency of $200 \mathrm{~Hz}$.

Table 1 The characteristics of the COP, COP + COG, and control groups

\begin{tabular}{lll}
\hline & $\operatorname{COP}(n=11)$ & $\operatorname{COP}+\operatorname{COG}(n=12)$ \\
\hline Age (years) & $23.2 \pm 2.3$ & $22.8 \pm 1.6$ \\
Sex & Male 6, female 5 & Male 8, female 4 $(n=11)$ \\
Height $(\mathrm{cm})$ & $165.7 \pm 6.9$ & $169.2 \pm 7.6$ \\
Body weight $(\mathrm{kg})$ & $56.8 \pm 7.5$ & $59.7 \pm 9.0$ \\
Foot length $(\mathrm{cm})$ & $24.2 \pm 1.8$ & $24.5 \pm 1.6$ \\
\hline
\end{tabular}


Twenty reflective markers were attached to the following bony landmarks: the acromioclavicular joint, the lateral epicondyle of the upper arm, the wrist, the head of the second metacarpal, the great trochanter of the femur, the lateral malleolus, the second metatarsal head, the calcaneus, and the $\mathrm{C}$, S1, and bilateral point of the external acoustic foramen. These markers were used to calculate the $\mathrm{COM}$ in the anteroposterior (AP) and mediolateral (ML) directions $\left(\mathrm{COM}_{\mathrm{AP}}\right.$ and $\left.\mathrm{COM}_{\mathrm{ML}}\right)$, based on the 14 body segments and an anthropometrical model [3]. A force plate (Kistler, Winterthur, Switzerland) was used to calculate the coordinates of the COP in the AP $\left(\mathrm{COP}_{\mathrm{AP}}\right.$; Appendix 1) and $\mathrm{ML}\left(\mathrm{COP}_{\mathrm{ML}}\right)$ directions. Force plate signals were collected at a sampling frequency of $1000 \mathrm{~Hz}$ and synchronized with the motion analysis system. The real-time $\mathrm{COG}$ in the AP direction $\left(\mathrm{COG}_{\mathrm{AP}}\right)$ was obtained from the force plate signals (Appendix 2) [10, 12].

Augmented visual feedback was provided in the form of fluctuating circles moving vertically upward as the $\mathrm{COP}_{\mathrm{AP}}$ and $\mathrm{COG}_{\mathrm{AP}}$ moved forward and downward as they moved backward. LabVIEW software (National Instruments, USA) was used to present this feedback on a screen (height $1.8 \mathrm{~m}$, width $2.5 \mathrm{~m}$ ) located approximately $5 \mathrm{~m}$ away from the participant. The vertical movement of the circles on the screen was 16 times greater than the true $\mathrm{COP}_{\mathrm{AP}}$ and $\mathrm{COG}_{\mathrm{AP}}$ displacements [16].

\section{Procedures}

The participants stood with both their feet placed on a foam pad (thickness $6.5 \mathrm{~cm}$, SAKAI Medical, Japan) throughout the pre-training, training, and post-training sessions. Only the AP direction was applied to reduce feedback complexity and allow the participants to focus on minimizing COP and COG fluctuations along a single axis [10]. The distance of the two horizontal lines on the screen corresponded to the two standard deviations (SD) of the first $\mathrm{COG}_{\mathrm{AP}}$ displacement measurement. The center point between the two lines identified the center of the force plate in the AP direction.

In the pre- and post-training test sessions (hereafter called pre-session and post-session, respectively), participants were required to lock their eyes on the fixed visual target and stand as steadily as possible for $60 \mathrm{~s}$. In the training session, participants in the COP group were required to align the center of the blank circle $(\varphi 13.5 \mathrm{~cm})$, which represented their $\mathrm{COP}_{\mathrm{AB}}$ to the center of the two horizontal lines for $40 \mathrm{~s}$ (Fig. 1a). The participants in the COP + COG group were required to align the center of the filled circle to the center of the horizontal lines and maintain an even distance in the up-down direction between the blank and filled circles $(\varphi 4.5 \mathrm{~cm})$, which respectively represented their $\mathrm{COP}_{\mathrm{AP}}$ and $\mathrm{COG}_{\mathrm{AB}}$ for $40 \mathrm{~s}$ (Fig. 1b). In practice, this required the participants to keep the filled circle inside the blank circle. The participants in
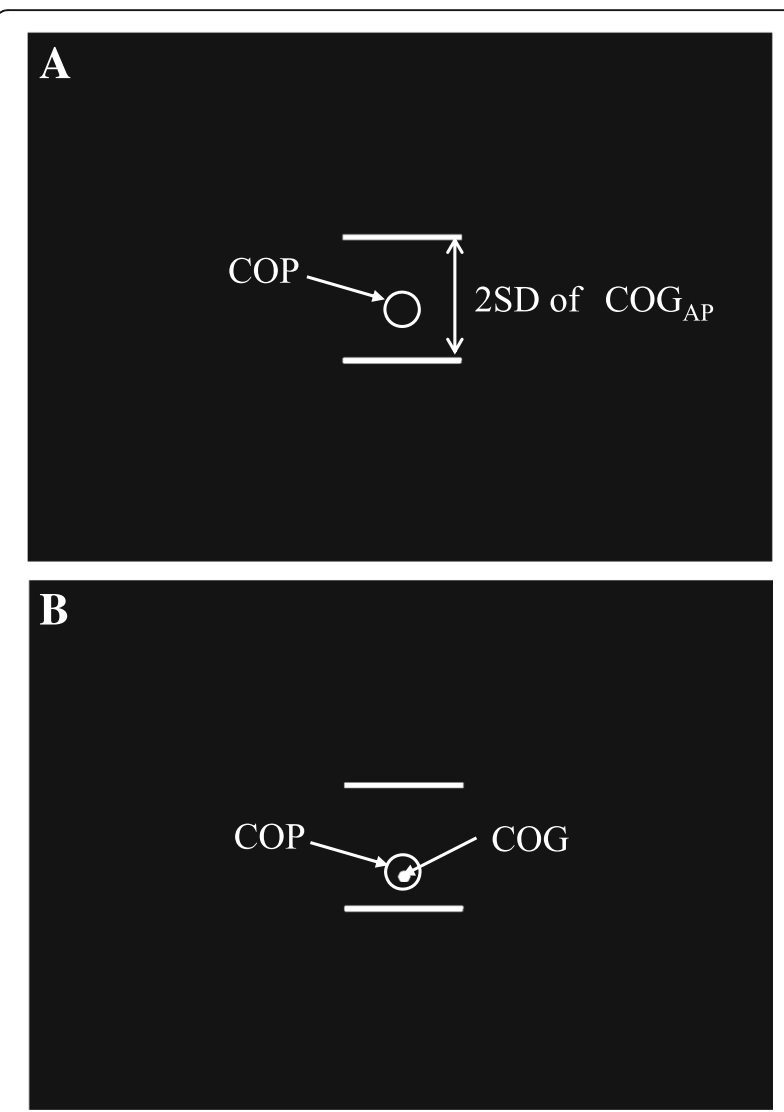

Fig. 1 Visual feedback on screen for the $\mathbf{a}$ COP group and $\mathbf{b}$ COP + COG group. Blank circles represent the real-time $\mathrm{COP}_{\mathrm{AP}}$, and filled circle represent the real-time $\mathrm{COG}_{\mathrm{AP}}$. These circles move in the vertical direction 16 times greater than the real amount of $\mathrm{COP}_{\mathrm{AP}}$ or $\mathrm{COG}_{\mathrm{AP}}$ movements. The width between the two horizontal lines indicates each participant's 2SD of $C G_{A P}$ displacements on a force plate during quiet standing

the control group were instructed to lock their eyes on a fixed visual target and stand as steadily as possible for $40 \mathrm{~s}$.

The participants were instructed to stand barefoot with their arms across their chest in front of a visual target located at an eye-level height on the screen. First, to measure the SD of the $\mathrm{COG}_{\mathrm{AP}}$ displacements, each participant was instructed to stand quietly with both feet placed together on the force plate with their eyes open for $10 \mathrm{~s}$. Horizontal lines, indicating the two SDs of each participant, were also projected on the screen during the training session (Fig. 1). Subsequently, the participants were required to stand on the foam pad, which was attached to the force plate with double-sided adhesive tapes, as steadily as possible with their feet placed together. The position of the feet on the pad was standardized: the center of the force plate in the sagittal plane was matched with the position of the feet $40 \%$ 
down the length from the heel [17]. The exact location where the feet were to be placed was marked on the pad to ensure that all the participants started with the same foot position in each trial. Each participant was asked to perform 12 trials with a 5-min rest after the first six trials. The break between trials was approximately $1 \mathrm{~min}$, while the time between the trials and pre- or post-sessions was $5 \mathrm{~min}$.

\section{Data and statistical analysis}

All signals were processed offline using MATLAB software (MathWorks, Natick, MA, USA). The motion analysis system data were filtered with a fourth-order $20-\mathrm{Hz}$ low-pass zero-lag Butterworth filter, and the force plate data were filtered with a fourth-order $8-\mathrm{Hz}$ low-pass zero-lag Butterworth filter. Although the signals obtained in the pre- and post-sessions had a duration of $60 \mathrm{~s}$, only 50-s durations of the signals were analyzed, excluding the initial and final $5 \mathrm{~s}$. The mean absolute velocities and root mean squares of both the $\mathrm{COP}$ and $\mathrm{COM}$ in the $\mathrm{AP}$ and $\mathrm{ML}$ directions $\left(\mathrm{COP}_{\mathrm{AP} / \mathrm{ML}}\right.$ velocity and $\mathrm{COM}_{\mathrm{AP} / \mathrm{ML}}$ velocity, $\mathrm{COP}_{\mathrm{AP} / \mathrm{ML}} \mathrm{RMS}$ and $\mathrm{COM}_{\mathrm{AP} / \mathrm{ML}}$ RMS) were calculated to assess postural stability. Furthermore, the mean absolute value of the COP - COM distance $\left(\mathrm{COP}-\mathrm{COM}_{\text {close }}\right.$, Eq. 1$)$ and the absolute value of the sum of the COP - COM distance $\left(\mathrm{COP}-\mathrm{COM}_{\text {even }}\right.$, Eq. 2) in the AP direction were calculated as follows:

$$
\begin{aligned}
& \mathrm{COP}-\mathrm{COM}_{\text {close }}=\frac{\sum_{t=1}^{N}\left|\mathrm{COP}_{t}-\mathrm{COM}_{t}\right|}{N} \\
& \mathrm{COP}-\mathrm{COM}_{\mathrm{even}}=\left|\sum_{t=1}^{N}\left(\mathrm{COP}_{t}-\mathrm{COM}_{t}\right)\right|
\end{aligned}
$$

where $N$ is the total sampling number. Thus, a lower value of $\mathrm{COP}-\mathrm{COM}_{\text {close }}$ indicated that movements of the COP were held closer to the COM in the AP direction. A shorter $\mathrm{COP}-\mathrm{COM}_{\text {even }}$ indicated that the $\mathrm{COP}$ position was more even, with fewer fluctuations around the $\mathrm{COM}$ in the AP direction. All parameters were normalized by the foot length (FL) of each participant.

Both one-way and two-way mixed-design ANOVA were used in each group (factor Group: COP, COP + COG, and control); one-way ANOVA was used to identify and analyze differences in biomechanical characteristics, and two-way mixed-design ANOVA compared the group data to test sessions (factor Test Session: pre and post) to analyze possible differences in the value of the indices. A post hoc analysis was performed using Bonferroni pairwise comparison, and Pearson's correlation coefficient was used to identify and analyze correlations between the $\mathrm{COM}_{\mathrm{AP}}$ velocity and COP - COM parameters. The statistical significance was set to $p<0.05$ for all tests.

\section{Results}

No significant differences were observed among the three groups in terms of age $\left(F_{2,31}=0.500, p=0.611\right)$, height $\left(F_{2,31}=1.170, p=0.324\right)$, weight $\left(F_{2,31}=0.391, p=0.680\right)$, and foot length $\left(F_{2,31}=1.779, p=0.186\right)$ (Table 1).

Figure 2 shows the characteristic data of $\mathrm{COM}_{\mathrm{AP}}$ and $\mathrm{COP}_{\mathrm{AP}}$ velocity profiles in the pre- and post-sessions for each group. In the COP + COG group, the amplitude of the $\mathrm{COM}_{\mathrm{AP}}$ velocity during the post-session decreased compared to the pre-session values, while the amplitude of the $\mathrm{COP}_{\mathrm{AP}}$ velocity did not change. Table 2 lists the mean and SD of velocities and RMSs of both the COM and $\mathrm{COP}$ in the AP and ML directions.

No significant effect was observed on the $\mathrm{COM}_{\mathrm{AP}}$ velocity between Group factors $\left(F_{2,31}=0.946, p=0.399\right)$. However, the $\mathrm{COM}_{\mathrm{AP}}$ velocity showed a significant change between Test session factors $\left(F_{1,31}=42.361, p<0.001\right)$. A significant interaction was observed between the Group and Test session factors in terms of the $\mathrm{COM}_{\mathrm{AP}}$ velocity $\left(F_{2,31}=3.391, p=0.047\right)$. The post hoc test revealed that post-session $\mathrm{COM}_{\mathrm{AP}}$ velocity values were significantly lower in the COP + COG group compared to those in the control group $(p=0.047)$. For both the COP $+\mathrm{COG}$ and control groups, the $\mathrm{COM}_{\mathrm{AP}}$ velocity in the post-session was significantly lower than that in the pre-session $(p<0.001)$; however, no significant difference was observed

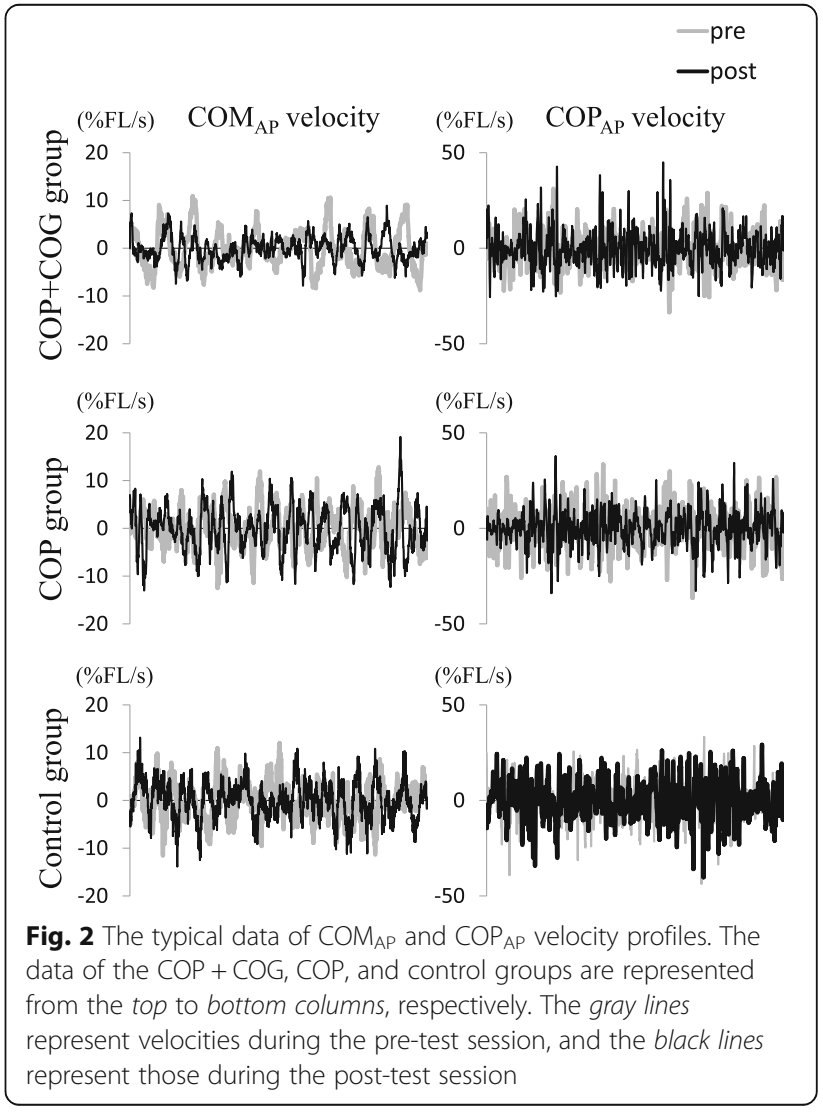


Table 2 The results of postural stability

\begin{tabular}{|c|c|c|c|c|c|c|}
\hline & \multicolumn{2}{|l|}{$\mathrm{COP}+\mathrm{COG}$} & \multicolumn{2}{|l|}{$\mathrm{COP}$} & \multicolumn{2}{|l|}{ Control } \\
\hline & Pre & Post & Pre & Post & Pre & Post \\
\hline $\mathrm{COM}_{\mathrm{AP}}$ velocity (\%FL/s) & $2.91 \pm 0.52^{*}$ & $2.27 \pm 0.41^{\dagger}$ & $2.94 \pm 0.58$ & $2.74 \pm 0.66$ & $3.02 \pm 0.41^{*}$ & $2.61 \pm 0.34$ \\
\hline $\mathrm{COM}_{\mathrm{AP}} \mathrm{RMS}(\% \mathrm{FL})$ & $3.24 \pm 0.72$ & $2.92 \pm 0.86^{\dagger}$ & $3.38 \pm 1.21$ & $2.86 \pm 0.62$ & $3.80 \pm 1.09$ & $4.01 \pm 1.25$ \\
\hline $\mathrm{COP}_{\text {AP velocity }(\% \mathrm{FL} / \mathrm{s})}$ & $8.40 \pm 2.06$ & $8.39 \pm 1.52$ & $9.26 \pm 2.97$ & $9.07 \pm 3.57$ & $8.95 \pm 1.66$ & $7.86 \pm 1.75$ \\
\hline $\mathrm{COP}_{\mathrm{AP}} \mathrm{RMS}(\% \mathrm{FL})$ & $3.96 \pm 0.73$ & $3.54 \pm 0.78$ & $4.12 \pm 1.13$ & $3.54 \pm 0.64$ & $4.33 \pm 0.95$ & $4.33 \pm 1.12$ \\
\hline $\mathrm{COM}_{\mathrm{ML}}$ velocity (\%FL/s) & $2.99 \pm 0.52$ & $2.76 \pm 0.34$ & $3.40 \pm 0.41^{*}$ & $3.12 \pm 0.59$ & $3.10 \pm 0.70$ & $2.78 \pm 0.38$ \\
\hline $\mathrm{COM}_{\mathrm{ML}} \mathrm{RMS}(\% \mathrm{FL})$ & $2.54 \pm 0.44$ & $2.59 \pm 0.49$ & $2.54 \pm 0.40^{*}$ & $3.11 \pm 0.67$ & $2.27 \pm 0.38$ & $2.59 \pm 0.60$ \\
\hline $\mathrm{COP}_{\mathrm{ML}}$ velocity (\%FL/s) & $9.12 \pm 2.51$ & $7.99 \pm 1.24$ & $10.48 \pm 3.02^{*}$ & $8.85 \pm 2.72$ & $9.48 \pm 2.59$ & $8.63 \pm 1.85$ \\
\hline $\mathrm{COP}_{\mathrm{ML}} \mathrm{RMS}(\% \mathrm{FL})$ & $3.43 \pm 0.47$ & $3.33 \pm 0.46$ & $3.61 \pm 0.44$ & $3.81 \pm 0.74$ & $3.25 \pm 0.54$ & $3.29 \pm 0.60$ \\
\hline
\end{tabular}

Mean \pm SD

${ }^{*} p<0.05$, between test sessions

${ }^{+} p<0.05$, compared to that of the control group in the post-session

in the COP group between the pre- and post-sessions $(p=0.117)$.

In terms of $\mathrm{COM}_{\mathrm{AP}}$ RMS, no significant main effect was observed for the factor Test session $\left(F_{1,31}=0.979\right.$, $p=0.330)$, nor was there a significant interaction $\left(F_{2,31}=\right.$ 1.036, $p=0.367)$. On the other hand, the $\mathrm{COM}_{\mathrm{AP}} \mathrm{RMS}$ showed a significant main effect for the factor Group $\left(F_{2,31}=4.158, p=0.025\right)$. The post hoc test revealed that the overall $\mathrm{COM}_{\mathrm{AP}}$ RMS of the COP + COG group was significantly lower than that of the control group $(p=0.042)$, while there was no significant difference between that of the COP and control groups $(p=0.068)$. The post-session $\mathrm{COM}_{\mathrm{AP}}$ RMS of the COP + COG group was significantly lower than that of the control group ( $p=0.022)$; however, there was no significant difference in the pre-session $(p=0.154)$.

In terms of the $\mathrm{COP}_{\mathrm{AP}}$ velocity and $\mathrm{COP}_{\mathrm{AP}} \mathrm{RMS}$, no significant differences were observed between the Group $\left(\mathrm{COP}_{\mathrm{AP}}\right.$ velocity $F_{2,31}=0.443, p=0.646 ; \mathrm{COP}_{\mathrm{AP}} \mathrm{RMS}$ $\left.F_{2}, \quad 31=2.199, \quad p=0.128\right)$ and Test session factors $\left(\mathrm{COP}_{\mathrm{AP}}\right.$ velocity $F_{1,31}=2.544, p=0.121 ; \mathrm{COP}_{\mathrm{AP}} \mathrm{RMS}$ $\left.F_{1}, 31=3.106, p=0.088\right)$. No significant interaction $\left(\mathrm{COP}_{\mathrm{AP}}\right.$ velocity $F_{2,31}=1.520, p=0.235 ; \mathrm{COP}_{\mathrm{AP}} \mathrm{RMS}$ $F_{2,31}=0.797, p=0.460$; Table 2) was observed.

With regard to $\mathrm{COM}_{\mathrm{ML}}$ velocity and $\mathrm{COM}_{\mathrm{ML}} \mathrm{RMS}$, no significant main effect was observed for the factor Group $\left(\mathrm{COM}_{\mathrm{ML}}\right.$ velocity $F_{2,31}=2.329, p=0.114 ; \mathrm{COM}_{\mathrm{ML}}$ RMS $F_{2,31}=2.842, p=0.074$ ), nor was there a significant interaction $\left(\mathrm{COM}_{\mathrm{ML}}\right.$ velocity $F_{2}, \quad 31=0.121, p=0.887$; $\mathrm{COM}_{\mathrm{ML}}$ RMS $\left.F_{2,31}=2.049, p=0.146\right)$. In contrast, the $\mathrm{COM}_{\mathrm{ML}}$ velocity and $\mathrm{COM}_{\mathrm{ML}}$ RMS showed significant main effects for the factor Test session $\left(\mathrm{COM}_{\mathrm{ML}}\right.$ velocity $F_{1,31}=12.497, p=0.001 ; \mathrm{COM}_{\mathrm{ML}}$ RMS $F_{1,31}=8.57$, $p=0.006)$. The post hoc test revealed that the postsession $\mathrm{COM}_{\mathrm{ML}}$ velocity of the COP group was significantly lower than that for the pre-session $(p=0.04)$. However, in the COP + COG and control groups, no significant difference was observed between the sessions (COP + COG group, $p=0.125$; control group, $p=0.056)$. Meanwhile, the post-session $\mathrm{COM}_{\mathrm{ML}}$ RMS of the COP group was significantly higher than that for the presession $(p=0.009)$. The $\mathrm{COM}_{\mathrm{ML}} \mathrm{RMS}$ of the COP + COG and control groups showed no significant difference between the sessions (COP + COG group, $p=0.82$; control group, $p=0.114$ ).

With regard to $\mathrm{COP}_{\mathrm{ML}}$ velocity, no significant main effect was observed for the factor Group $\left(F_{2}, 31=0.722, p=\right.$ $0.494)$, nor was there a significant interaction $\left(F_{2}, 31=0.526\right.$, $p=0.596)$. On the contrary, the $\mathrm{COP}_{\mathrm{ML}}$ velocity presented a significant main effect for the factor Test session $\left(F_{1,31}=\right.$ $15.86, p<0.001)$. The post hoc test revealed that the postsession $\mathrm{COP}_{\mathrm{ML}}$ velocity of the COP group was significantly lower than that for the pre-session $(p<0.001)$. However, in the COP + COG and control groups, no significant difference was found between the sessions (COP + COG group, $p=0.081$; control group, $p=0.195$ ).

Meanwhile, the $\mathrm{COP}_{\mathrm{ML}}$ RMS demonstrated no significant differences between the Group $\left(F_{2,31}=2.697, p=0.083\right)$ and Test session factors $\left(F_{1,31}=0.24, p=0.628\right)$. No significant interaction $\left(F_{2,31}=0.789, p=0.463\right)$ was observed.

No significant differences were observed between the Group $\left(F_{2,31}=2.162, p=0.132\right)$ or Test session factors $\left(F_{1,31}=0.047, p=0.829\right)$ in terms of COP $-\mathrm{COM}_{\text {even }}$. A significant $\mathrm{COP}-\mathrm{COM}_{\text {even }}$ interaction was observed between the two factors $\left(F_{2}, \quad 31=3.517, p=0.042\right.$; Fig. 3a): The post hoc test revealed that the postsession COP $-\mathrm{COM}_{\text {even }}$ was significantly smaller for the COP + COG group than that for the pre-session $(p=$ $0.002)$. On the other hand, no significant difference was observed between the pre- and post-sessions in the COP group $(p=0.234)$. Furthermore, the $\mathrm{COP}-\mathrm{COM}_{\text {even }}$ for the COP + COG group was significantly smaller than that for the COP group $(p<0.001)$ or control group $(p=0.033)$ in the post-session. 


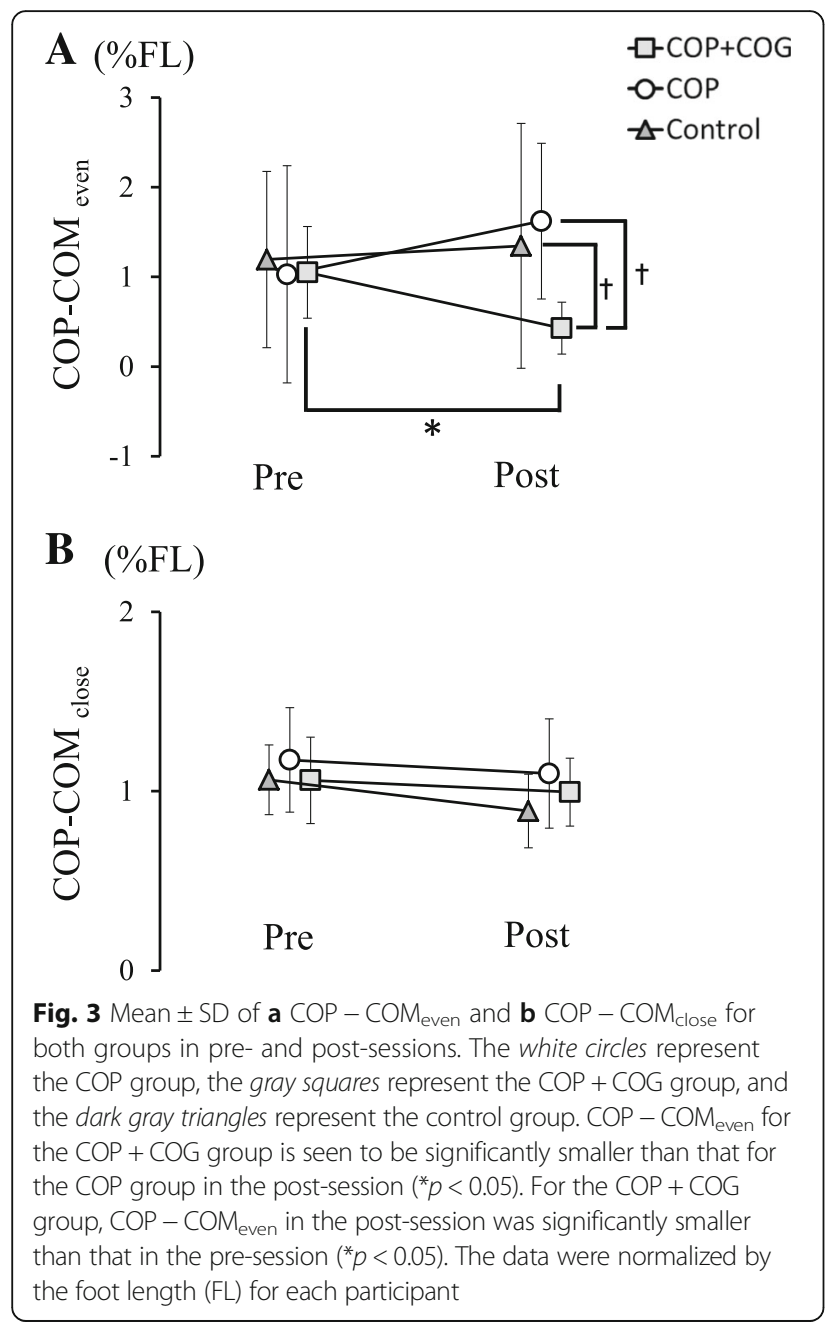

In terms of $\mathrm{COP}-\mathrm{COM}_{\text {close }}$, no significant effects were observed for the factor Group $\left(F_{2}, 31=1.457, p=0.248\right)$ and no significant interaction $\left(F_{2}, 31=1.267, p=0.296\right)$ was observed. On the other hand, the factor Test session showed significant discrepancies $\left(F_{1,31}=11.884, p=0.002\right.$; Fig. 3b).

In the post-session, significant positive correlations were observed between the COM velocity and $\mathrm{COP}-\mathrm{COM}_{\text {even }}$ in the COP + COG group $(r=0.729, p<0.01)$ and the COP group $(r=0.526, p<0.05)$. Significant positive correlations were observed between the COM velocity and COP $-\mathrm{COM}_{\text {close }}$ in the COP + COG group $(r=0.823$, $p<0.01)$ and the COP group $(r=0.843, p<0.01)$. No significant correlations were observed between the COM velocity and COP $-\mathrm{COM}_{\text {even }}(r=-0.291, p>0.05)$ or COP - $\mathrm{COM}_{\text {close }}(r=0.457, p>0.05)$ in the control group. Significant positive correlations were observed between the COM velocity and COP $-\mathrm{COM}_{\text {even }}(r=0.670$, $p<0.01$ : Fig. 4a) as well as COP $-\mathrm{COM}_{\text {close }}(r=0.798, p<$
0.01: Fig. 4b) for the combined data of the COP + COG and COP groups.

\section{Discussion}

The mean absolute velocity of the COM has been proven to be a highly reliable and sensitive indicator of postural sway $[18,19]$. The main finding of this study is that the $\mathrm{COM}_{\mathrm{AP}}$ velocity decreased after the training session in the COP + COG and control groups, but not in the COP group. The $\mathrm{COM}_{\mathrm{AP}}$ velocity in the COP + COG group was lower than that in the control group during post-session quiet standing (Table 2). Furthermore, the $\mathrm{COM}_{\mathrm{AP}}$ RMS was significantly lower in the COP + COG group compared to that in the control group following training. These results suggest that training conducted using simultaneous $\mathrm{COP}_{\mathrm{AP}}$ and $\mathrm{COG}_{\mathrm{AP}}$ visual feedback increases postural stability compared to training using the $\mathrm{COP}_{\mathrm{AP}}$ alone under a no-feedback condition. Therefore, the results of this study have confirmed the hypothesis.

The effects that were related to a decrease in the postsession $\mathrm{COM}_{\mathrm{AP}}$ velocity would be enhanced by even

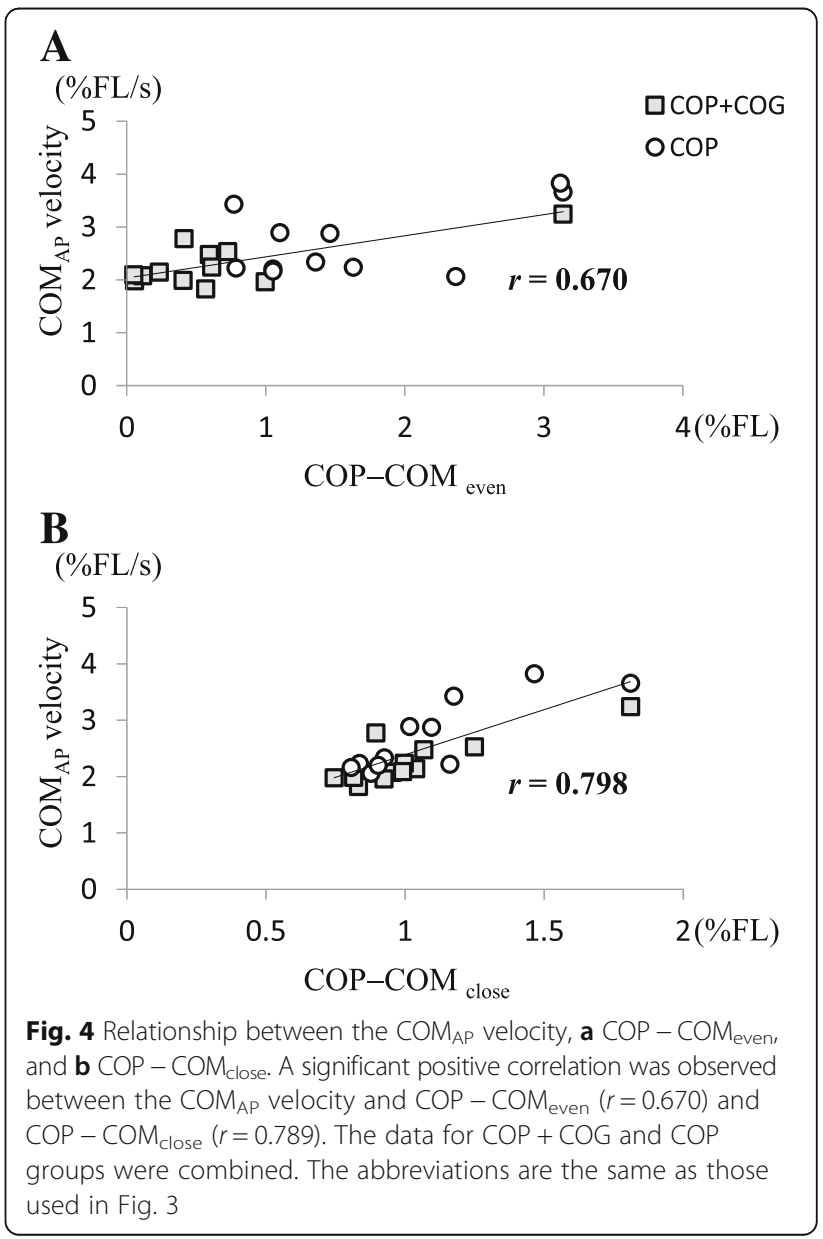


fluctuations of the $\mathrm{COP}_{\mathrm{AP}}$ around the $\mathrm{COG}_{\mathrm{AP}}$ (Fig. 3a) because of the significant correlation between the $\mathrm{COM}_{\mathrm{AP}}$ velocity and COP - $\mathrm{COM}_{\text {even }}$ (Fig. 4a). According to the inverted pendulum model, inertial forces produced by even fluctuations of the COP toward the COG restrain COM movements toward the center of its fluctuation range; this is because the COP - COM distance reflects the moment arm for inertial forces, such as propulsion toward the COM or braking against movements toward the COM $[20,21]$.

Interestingly, the $\mathrm{COP}_{\mathrm{AP}}$ velocity did not decrease even though the $\mathrm{COM}_{\mathrm{AP}}$ velocity decreased in the postsession for each group (Table 2). In general, minimizing the COM displacement would be expected to result in a concurrent decrease in the COP displacement [22]. However, Carpenter et al. [23] and Murnaghan et al. [24] showed that COP displacements increased when COM movements were stabilized. They proposed that COP fluctuations played an exploratory role, gathering sensory information during quiet standing. The results of this study indicate that a decrease in the $\mathrm{COM}_{\mathrm{AP}}$ velocity do not result in a concurrent decrease in the $\mathrm{COP}_{\mathrm{AP}}$ velocity; as such, the $\mathrm{COP}$ and $\mathrm{COM}$ velocities may realistically behave in different ways.

No significant differences of the velocity and the RMS of COM and COP between the pre- and post-sessions were found in the COP + COG group in the ML direction. Therefore, the decreased postural stability in the ML direction after the training could not be confirmed in the $\mathrm{COP}+\mathrm{COG}$ group. Interestingly, the $\mathrm{COM}_{\mathrm{ML}}$ and $\mathrm{COP}_{\mathrm{ML}}$ velocities in the $\mathrm{COP}$ group decreased after the training. However, the increased postural stability in the ML direction could not be confirmed because the $\mathrm{COM}_{\mathrm{ML}}$ RMS in the COP group increased after the training. The standing postural controls for the AP or ML direction are involved in two distinct ankle and hip mechanisms [3]. The possibility effects of the postural control during quiet standing between the two mechanisms by the feedback training should be further investigated in future studies [25].

We suspect that efforts to maintain the $\mathrm{COP}_{\mathrm{AP}}$ at an even distance from the $\mathrm{COG}_{\mathrm{AP}}$ may have indirectly contributed to reducing the $\mathrm{COP}-\mathrm{COM}$ distance; however, no interaction was observed in terms of $\mathrm{COP}-\mathrm{COM}_{\text {close }}$. The quantitative results of this study showed that the $\mathrm{COM}_{\mathrm{AP}}$ velocity was correlated to $\mathrm{COP}-\mathrm{COM}_{\text {close }}$ (Fig. 4b). Therefore, adding visual targets indicating the $\mathrm{COP}_{\mathrm{AP}}$ and $\mathrm{COG}_{\mathrm{AP}}$ (e.g., two other horizontal lines positioned along the centers of the blank circle representing the $\mathrm{COP}_{\mathrm{AP}}$ and the filled circle representing the $\mathrm{COG}_{\mathrm{AP}}$ ) and requiring participants to reduce the distance between these two horizontal lines may also be effective to decelerate the $\mathrm{COM}_{\mathrm{AP}}$ velocity.
The limitation of this study is that experiments were performed with a small sample size of participants. In addition, the adaptation effects of training may not be detected sufficiently with the small amount of training the participants underwent. Therefore, the training effects in the COP group may not be detected, although the $\mathrm{COM}_{\mathrm{AP}}$ velocity in the control group decreased after training. Furthermore, the force under the feet may not be identical to that under the foam pad because the force or moment could spread in the pad. The learning effects of this novel balance training should be further investigated with a retention test and applied to individuals with postural instability in future studies.

\section{Conclusion}

Simultaneous visual feedback training that uses both the $\mathrm{COP}_{\mathrm{AP}}$ and $\mathrm{COG}_{\mathrm{AP}}$ and focuses on their interaction, reduces postural sway during quiet standing better than the training designed to affect only the $\mathrm{COP}_{\mathrm{AP}}$ under the no-feedback condition. It can therefore be stated that even $\mathrm{COP}_{\mathrm{AP}}$ fluctuations around the $\mathrm{COG}_{\mathrm{AP}}$ would be effective for maintaining postural static balance through an associated reduction in $\mathrm{COM}_{\mathrm{AP}}$ velocity.

\section{Appendix 1}

The $\mathrm{COP}_{\mathrm{AP}}$ was calculated as follows:

$$
\mathrm{COP}_{\mathrm{AP}}=\frac{F y \times\left(-D_{0}-D_{\text {mat }}\right)+M x}{F z}
$$

where $F y$ and $F z$ denote the force in the AP and vertical directions, respectively. $M x$ denotes the moment around the lateral axis. $D_{0}$ and $D_{\text {mat }}$ denote the thickness of the force plate and the foam pad, respectively.

\section{Appendix 2}

If human posture during quiet standing can be approximated as an inverted pendulum, the equation of motion can be described as follows:

$$
I \ddot{\theta}=m g h \sin \theta-T
$$

where $m$ is the mass of the body excluding feet, $g$ is the gravitational acceleration coefficient, $h$ is the distance between COM and the ankle joint, $I$ is the moment of inertia of the body about the ankle joint, $\theta$ is the body angle to vertical axis, $\ddot{\theta}$ is the body angular acceleration, and $T$ is the ankle torque produced about the participant's ankle joint. Assuming that the body sway amplitude is small, $T \approx m g C O P$ and $m g h \sin \theta m g C O G$. Thus, the right-hand side of Eq. (3) can be rewritten as $-m g(\mathrm{COP}-\mathrm{COG})$. Considering an additional approximation of $\ddot{\theta} \approx C \ddot{O} M / h=A C C / h=F_{Y} / m h$, Eq. (3) can be derived as follows: 


$$
\mathrm{COG}_{\mathrm{AP}}=\mathrm{COP}_{\mathrm{AP}}-\left(\frac{1}{m g h}\right) * \frac{F y}{m^{\prime}}
$$

where $F y$ is the force in the AP direction.

$$
\begin{aligned}
& m=0.971 \mathrm{M}, \\
& h=0.547 \mathrm{H}, \\
& I=0.319 \mathrm{MH}^{2},
\end{aligned}
$$

where $\mathrm{M}$ is the participant mass of body in kilograms and $\mathrm{H}$ is the participant height in meters.

The details of the derivation steps and the anthropometric estimation of body dimensions can be obtained from previous reports $[3,12]$.

\section{Funding}

This work was supported in part by a Japanese Grant-in-Aid for Scientific Research (25350747, 16K16420).

\section{Availability of data and materials}

The datasets during and/or analyzed during the current study are available from the corresponding author on reasonable request.

\section{Authors' contributions}

TA, KT, and HMan designed the study. TA and HMae supervised the project. KT, HMan, NH, YS, and ST recorded, analyzed, and interpreted the data. TA and $\mathrm{KT}$ wrote the manuscript. All authors read and approved the final manuscript.

\section{Ethics approval and consent to participate}

All participants gave written informed consent after a complete explanation of this study. This study was approved by the Institutional Review Board, Faculty of Health Sciences of Hokkaido University.

\section{Consent for publication}

All participants gave written informed consent for publication after a complete explanation of this study.

\section{Competing interests}

The authors declare that they have no competing interests.

\section{Publisher's Note}

Springer Nature remains neutral with regard to jurisdictional claims in published maps and institutional affiliations.

\section{Author details}

${ }^{1}$ Graduate School of Health Sciences, Hokkaido University, N12-W5, Kita-ku, Sapporo, Hokkaido 060-0812, Japan. ²Department of Rehabilitation Science, Faculty of Health Sciences, Hokkaido University, N12-W5, Kita-ku, Sapporo, Hokkaido 060-0812, Japan.

Received: 20 December 2016 Accepted: 4 July 2017 Published online: 19 July 2017

\section{References}

1. Shumway-Cook A, Wollacott M. Motor control: translating research into clinical practice. 4th ed. Philadelphia: Lippincott Williams \&Wilkins; 2011.

2. Riach $\mathrm{CL}$, Starkes JL. Stability limits of quiet standing postural control in children and adults. Gait Posture. 1993:1:105-11.

3. Winter DA. Biomechanics and motor control of human movement. 4th ed Hoboken: Wiley; 2009

4. Dault MC, de Haart M, Geurts ACH, Arts IMP, Nienhuis B. Effects of visual center of pressure feedback on postural control in young and elderly healthy adults and in stroke patients. Hum Mov Sci. 2003;22:221-36.
5. Zijlstra A, Mancini M, Chiari L, Zijlstra W. Biofeedback for training balance and mobility tasks in older populations: a systematic review. J Neuroeng Rehabil. 2010;7:58

6. Halicka Z, Lovotkova J, Buckova K, Hlavacka F. Effectiveness of different visual biofeedback signals for human balance improvement. Gait Posture. 2014;39:410-4.

7. Geiger RA, Allen JB, O'Keefe J, Hicks RR. Balance and mobility following stroke: effects of physical therapy interventions with and without biofeedback/forceplate training. Phys Ther. 2001;81:995-1005.

8. Freitas SMSF, Duarte M. Joint coordination in young and older adults during quiet stance: effect of visual feedback of the center of pressure. Gait Posture. 2012;35:83-7.

9. Kilby MC, Slobounov SM, Newell KM. Augmented feedback of COM and COP modulates the regulation of quiet human standing relative to the stability boundary. Gait Posture. 2016;47:18-23.

10. Lakhani B, Mansfield A. Visual feedback of the centre of gravity to optimize standing balance. Gait Posture. 2015:41:499-503.

11. Corriveau H, Hebert R, Prince F, Raiche M. Postural control in the elderly: an analysis of test-retest and interrater reliability of the COP-COM variable. Arch Phys Med Rehab. 2001;82:80-5.

12. Masani K, Vette AH, Kouzaki M, Kanehisa H, Tukunaga T, Popovic MR. Larger center of pressure minus center of gravity in the elderly induces larger body acceleration during quiet standing. Neurosci Lett. 2007;422:202-6.

13. Yu E, Abe M, Masani K, Kawashima N, Eto F, Haga N, Nakazawa K. Evaluation of postural control in quiet standing using center of mass acceleration: comparison among the young, the elderly, and people with stroke. Arch Phs Med Rehabil. 2008;89:1133-9.

14. Mani H, Hsiao SF, Takeda K, Hasegawa N, Tozuka M, Tsuda A, Ohashi T, Suwahara T, Ito K, Asaka T. Age-related changes in distance from center of mass to center of pressure during one-leg standing. J Mot Behav. 2015:47:282-90.

15. Ibuki A, Mani H, Takeda K, Hasegawa N, Yamamoto K, Maejima H, Asaka T: Characteristic relationship between the centre of pressure and the centre of mass during quiet standing in female ballet dancers. Int Phys Med Rehab J 2017; DOI: 10.15406/ipmrj.2017.01.00009

16. Cawsey RP, Cha R, Carpenter MG, Sanderson DJ. To what extent can increasing the magnification of visual feedback of the centre of pressure position change the control of quiet standing balance? Gait Posture. 2009;29:280-4.

17. Okuni I, Uchi M, Harada T. Sagittal-plane spinal curvature and center of foot pressure in healthy young adults. J Med Soc Toho Univ. 2006:53:254-60.

18. Raymakers JA, Samson MM, Verhaar HJ. The assessment of body sway and the choice of the stability parameter(s). Gait Posture. 2005;21:48-58.

19. van Dieen $\mathrm{JH}$, Koppes LL, Twisk JW. Postural sway parameters in seated balancing; their reliability and relationship with balancing performance. Gait Posture. 2010:31:42-6.

20. Chang H, Krebs DE. Dynamic balance control in elders: gait initiation assessment as a screening tool. Arch Phs Med Rehabil. 1999:80:490-4.

21. Jian $Y$, Winter DA, Ishac MG, Gilchrist L. Trajectory of the body COG and COP during initiation and termination of gait. Gait Posture. 1993:1:9-22.

22. Horak FB, MacPherson JM. Postural orientation and equilibrium. In: Rowell LB, Shepherd JT, editors. Handbook of physiology, section 12, exercise: regulation and Integration of Multiple Systems. New York: Oxford University Press; 1996. p. 255-92.

23. Carpenter MG, Murnaghan CD, Inglis JT. Shifting the balance: evidence of an exploratory role for postural sway. Neuroscience. 2010;171:196-204.

24. Murnaghan CD, Horslen BC, Inglis JT, Carpenter MG. Exploratory behaviour during stance persists with visual feedback. Neuroscience. 2011;195:54-9.

25. Rougier PR. Undisturbed stance control in healthy adults is achieved differently along anteroposterior and mediolateral axes: evidence from visual feedback of various signals from center of pressure trajectories. J Mot Behav. 2009:41:197-206. 